\title{
Impact of post-sepsis cardiovascular complications on mortality in sepsis survivors: a population-based study
}

Meng-Huan Wu', Po-Yang Tsou², Yu-Hsun Wang ${ }^{2}$, Meng-tse Gabriel Lee ${ }^{3}$, Christin Chih Ting Chao ${ }^{4}$, Wan-Chien Lee ${ }^{3}$, Si-Huei Lee ${ }^{5,6}$, Jiun-Ruey Hu², Jiunn-Yih Wu ${ }^{7}$, Shy-Shin Chang ${ }^{8}$ and Chien-Chang Lee ${ }^{3,9^{*}}$ (i)

\begin{abstract}
Background: It remains unclear whether sepsis-related cardiovascular complications have an adverse impact on survival independent of pre-existing comorbidities. To investigate the survival impact of post-sepsis cardiovascular complications among sepsis survivors, we conducted a population-based study using the National Health Insurance Database of Taiwan.

Methods: We identified sepsis patients from the National Health Insurance Research Database of Taiwan using ICD9-CM codes involving infection and organ dysfunction between 2000 and 2011. Post-sepsis incident myocardial infarction (MI) and stroke were ascertained by ICD-9-CM codes and antiplatelet treatment. We constructed a nonsepsis comparison cohort using propensity score matching to ascertain the association between sepsis and cardiovascular complications. Furthermore, we compared the 180-day mortality and 365-day mortality between patients surviving sepsis with or without post-sepsis Ml or stroke within 70 days of hospital discharge. We constructed Cox regression models adjusting for pre-existing comorbidities to evaluate the independent survival impact of post-sepsis Ml or stroke among sepsis survivors.
\end{abstract}

Results: We identified 42,316 patients hospitalized for sepsis, from which we matched 42,151 patients 1:1 with 42, 151 patients hospitalized without sepsis. Compared to patients hospitalized without sepsis, patients hospitalized with sepsis had an increased risk of Ml or stroke (adjusted odds ratio 1.72, 95\% Cl 1.60-1.85). Among 42,316 patients hospitalized for sepsis, 486 (1.15\%) patients developed incident stroke and 108 (0.26\%) developed incident MI within 70 days of hospital discharge. Compared to sepsis survivors without cardiovascular complications, sepsis survivors with incident $\mathrm{Ml}$ or stroke had a higher mortality rate at 180 days $(11.68 \%$ vs. $4.44 \%, P=0.003)$ and at 365 days $(16.75 \%$ vs. $7.11 \%, P=0.005)$. Adjusting for age, sex, and comorbidities, post-sepsis Ml or stroke was independently associated with increased 180-day (adjusted hazard ratio [HR] 2.16, 95\% Cl 1.69-2.76) and 365-day (adjusted HR 1.90, 95\% Cl 1.54-2.32) mortality.

Conclusions: Compared to sepsis patients without incident Ml or stroke, sepsis patients with incident Ml or stroke following hospital discharge had an increased risk of mortality for up to 365 days of follow-up. This increased risk cannot be explained by pre-sepsis comorbidities.

Keywords: Survival analysis, Post-sepsis myocardial infarction, Post-sepsis stroke

\footnotetext{
* Correspondence: cclee100@gmail.com

${ }^{3}$ Department of Emergency Medicine, National Taiwan University Hospital,

No.7, Chung Shan S. Rd., Zhongzheng Dist., Taipei 100, Taiwan

${ }^{9}$ Department of Emergency Medicine, Health Data Science Research Group,

National Taiwan University Hospital, No.7, Chung Shan S. Rd., Zhongzheng

Dist., Taipei 100, Taiwan

Full list of author information is available at the end of the article
}

(c) The Author(s). 2019 Open Access This article is distributed under the terms of the Creative Commons Attribution 4.0 International License (http://creativecommons.org/licenses/by/4.0/), which permits unrestricted use, distribution, and reproduction in any medium, provided you give appropriate credit to the original author(s) and the source, provide a link to the Creative Commons license, and indicate if changes were made. The Creative Commons Public Domain Dedication waiver (http://creativecommons.org/publicdomain/zero/1.0/) applies to the data made available in this article, unless otherwise stated. 


\section{Background}

Sepsis, which refers to life-threatening organ dysfunction caused by a dysregulated host response to infection, is a major public health concern [1]. In Taiwan, the incidence of sepsis has steadily risen from 638 per 100,000 persons to 772 per 100,000 persons from 2002 to 2012, while the annual mortality has steadily decreased from 27.8 to $22.8 \%$ over the same time period [2]. While short-term sepsis mortality is decreasing, mid- to longterm sepsis mortality has remained high as many patients die in the subsequent months. Recent studies have suggested that the increased risk of mid- to long-term mortality after sepsis cannot be explained by the preexisting comorbid conditions before sepsis and might be attributed to increased post-sepsis cardiovascular complications instead [3]. Sepsis increases the risks of cardiovascular complications during and shortly after admission, with up to fourfold increase in the risk [4-7]. The increased risk of cardiovascular complications has been attributed to a variety of pathophysiologic mechanisms, including immunoparalysis, depression of ventricular function, arrhythmia, organ ischemia related to increased oxygen demand, procoagulant changes in the blood, impaired cardiovascular autonomic response, and accelerated atherosclerosis [8-10]. Consistent with prior literature, our previous work using the National Health Insurance Research Database of Taiwan demonstrated that patients with sepsis are at markedly elevated risk of incident $\mathrm{MI} /$ stroke during the first 70 days after hospital discharge [11]. We found that after this critical 70-day period, sepsis survivors have a comparable risk of post-sepsis $\mathrm{MI} /$ stroke with non-sepsis control patients.

Despite the established association between sepsis and cardiovascular complications, limited information is known about the survival impact of these post-sepsis cardiovascular complications. Among the difficulties in assessing the survival impact of post-sepsis cardiovascular complications are the rarity of post-sepsis cardiovascular complications and the complex competing risk relationship between mortality and post-sepsis incident cardiovascular complications [12, 13]. The aim of this study was twofold. First, we sought to determine the independent association of sepsis with incident cardiovascular disease after sepsis hospitalization with the construction of a non-sepsis comparison group. Second, we sought to ascertain if the increase in mortality associated with cardiovascular events in sepsis patients exceeded the mortality associated with cardiovascular events in non-sepsis patients. The use of a national population-based database allows us to build a large sepsis cohort with a sufficient number of events to compare the outcome between patients with and without incident $\mathrm{MI} /$ stroke after sepsis $[14,15]$.

\section{Methods}

\section{Data source}

The present study used the National Health Insurance Research Database (NHIRD) of Taiwan. Taiwan's National Health Insurance (NHI) program is a single-payer mandatory health insurance system that covers over $99 \%$ of the 23,000,000 people residing in Taiwan. The database used a systematic sampling strategy to select one million participants who were representative of the demographic and geographic region distribution of Taiwan in 2001. This sample, hereafter referred to as the one million longitudinal sample, was followed from 2001 to 2012 to form a closed cohort for research purposes. The database collects outpatient and inpatient electronic records on demographics, eligibility, vital status, diagnoses (International Classification of Diseases, ninth revision, Clinical Modification [ICD-9-CM]), operations, and prescriptions. All claims can be linked in chronological order to provide a temporal sequence of all health services utilization. Patient consent was not required for this study as this was an anonymized electronic database study. This study was approved by the Institutional Review Board of National Taiwan University Hospital. This observational study was performed in accordance with the Strengthening the Reporting of Observational Studies in Epidemiology (STROBE) guidelines for reporting observational studies [16].

\section{Design and study participants}

The study was a population-based cohort study, consisting of all emergency department (ED) or hospital-treated sepsis patients between 2001 and 2011.

\section{Identification of sepsis cases}

Sepsis patients were identified based on the coding system used by Angus et al., which is considered to most closely resemble the latest Sepsis-3 definition among the published algorithms [17]. Operationally, sepsis patients were identified using ICD-9-CM codes for the presence of either a bacterial or fungal infection (Additional file 4: Appendix 1) plus dysfunction of one or more organ systems (Additional file 4: Appendix 2) [1]. The ICD-9-CM codes for the identification of infections in this study were the same as those used by Angus et al. (including 1286 distinct infection codes). The ICD-9-CM codes for acute organ dysfunctions were supplemented with $\mathrm{NHI}$ procedure codes to increase specificity. In this manuscript, MI refers to myocardial infarction alone, stroke refers to cerebrovascular events alone, and $\mathrm{MI} /$ stroke stands for the combined outcome of MI and stroke.

\section{Construction of the non-sepsis comparison cohort}

To ascertain the independent association between sepsis and incident myocardial infarction (MI)/stroke after 
sepsis hospitalization, we constructed a non-sepsis comparison cohort using the risk set sampling and propensity score matching technique. The non-sepsis comparison cohort was constructed using a two-stage procedure. In the first stage, we used the risk set sampling method to sample 100 non-sepsis patients for each sepsis case, matching on admission date, 5-year age group, sex, and quartile Charlson Comorbidity Score (0, 1-2, 3-4 and $\geq 5$ ) [18]. In the second stage, we created a propensity score (PS) consisting of a comprehensive set of covariates associated with sepsis (Additional file 1: Table S1). We performed 1:1 PS matching using a greedy algorithm to construct the final non-sepsis comparison cohort. The cohort construction process is shown in Fig. 1.
Identification of post-sepsis cardiovascular complications As the primary aim was to analyze the impact of postsepsis stroke/MI on mid- or long-term survival, the primary "exposure" in this study was incident $\mathrm{MI} /$ stroke after sepsis hospitalization. Our previous study found that the critical period for $\mathrm{MI} /$ stroke is 70 days after discharge from a sepsis hospitalization. We therefore assessed for $\mathrm{MI} /$ stroke within the 70 -day risk period as the primary exposure of this study. Patients with stroke were identified by the presence of an ICD-9-CM diagnosis code of 433.xx or 434.xx, which have a positive predictive value (PPV) of 0.96 and 1.00 , respectively, in the NHIRD database [19]. Patients with incident acute MI were identified by any primary or secondary

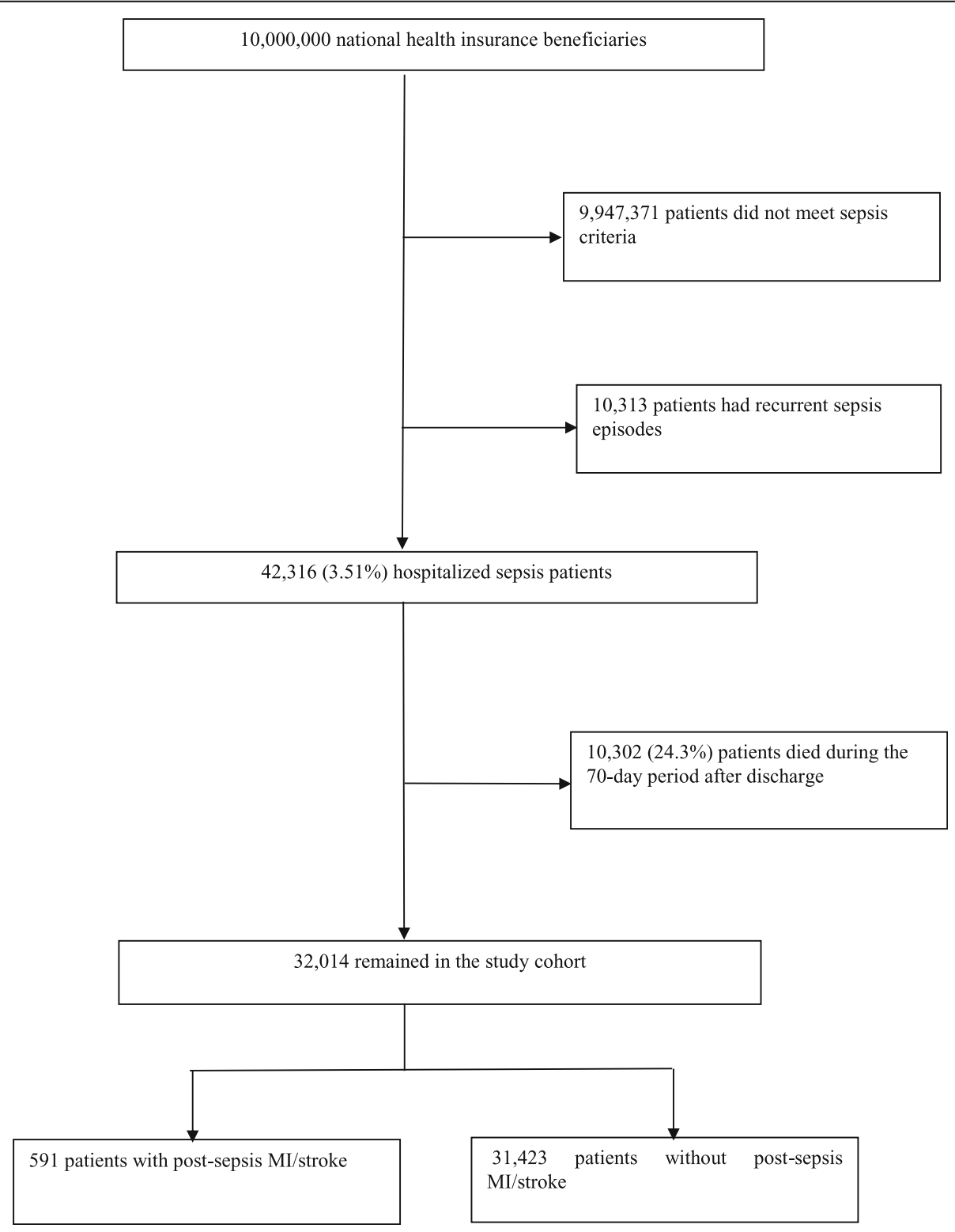

Fig. 1 Construction of the study cohort 
admission diagnosis containing ICD-9-CM code of 410.xx together with a prescription for antiplatelet therapy, such as aspirin, clopidogrel, dipyridamole, and ticlopidine. Newer classes of antiplatelets were not available in Taiwan during the study period. The above search algorithm yields a PPV of 0.84 for acute MI in the NHIRD database [20]. The primary outcomes of interest were 180-day and 365-day all-cause mortality.

\section{Follow-up of cohort}

For both the primary sepsis cohort and non-sepsis comparison cohort, we anchored the 71st day after hospital discharge as the index day of cohort entry. Patients were followed up for three outcomes: death, termination of health insurance coverage, and end of study (the 365th day from the index date), whichever came first. To control for unbalanced covariates between patients with/ without post-sepsis cardiovascular events, we collected information on demographics, urbanization level, insurance premium level, chronic comorbidities, and risk factors for sepsis. In order to remove the effect of covariates that could develop after incident cardiovascular events, we collected all covariate information from the beginning of the study (year 2001) to the discharge date of the index hospitalization. The level of urbanization of the cities/towns was stratified into four levels based upon a composite score obtained by calculating population density (people $/ \mathrm{km}^{2}$ ), population percent of people with an educational level of college or above (\%), percent of people over 65 years (\%), percent of agriculture workers (\%), and the number of physicians per 100,000 people. To further control for general health conditions that are not reflected by ICD-9 CM codes [21], we further collected the frequency of healthcare facility utilization in the 1-year period before sepsis admission as a proxy indicator of general health. The timeline of the study design and periods of data collection can be seen in Fig. 2.

\section{Data analysis}

The analysis was implemented in two stages. In the primary analysis, we analyzed the impact of incident MI/ stroke on the mid- or long-term outcome of sepsis survivors. In the secondary analysis, we compared the incidence and outcome of incident stoke/MI after hospital discharge between the sepsis and non-sepsis cohorts. In the primary analysis, we first compared the baseline characteristics between sepsis survivors who developed incident acute $\mathrm{MI} /$ stroke and those who did not develop incident acute $\mathrm{MI} /$ stroke. Categorical variables were presented as frequency and percentage and compared using chi-squared tests. Continuous variables were presented as mean or median and compared by non-parametric Mann-Whitney $U$ tests. We then plotted the cumulative mortality curves of patients with/without post-sepsis $\mathrm{MI} /$ stroke. The differences in cumulative mortality between the groups were compared using the Wilcoxon signed-rank test. The survival impact of post-sepsis MI/ stroke was assessed by multivariable Cox regression analyses. The proportional hazards assumption was checked by plotting curves of the $\log$ of the negative log of the survival function against log time for patients with/without post-sepsis $\mathrm{MI} /$ stroke. Potential confounders were determined based on the potential relevance given the literature, including the following variables: demographics, urbanization level, insurance premium, comorbidity, and healthcare service utilization. In the secondary analysis, we constructed a non-sepsis comparison cohort

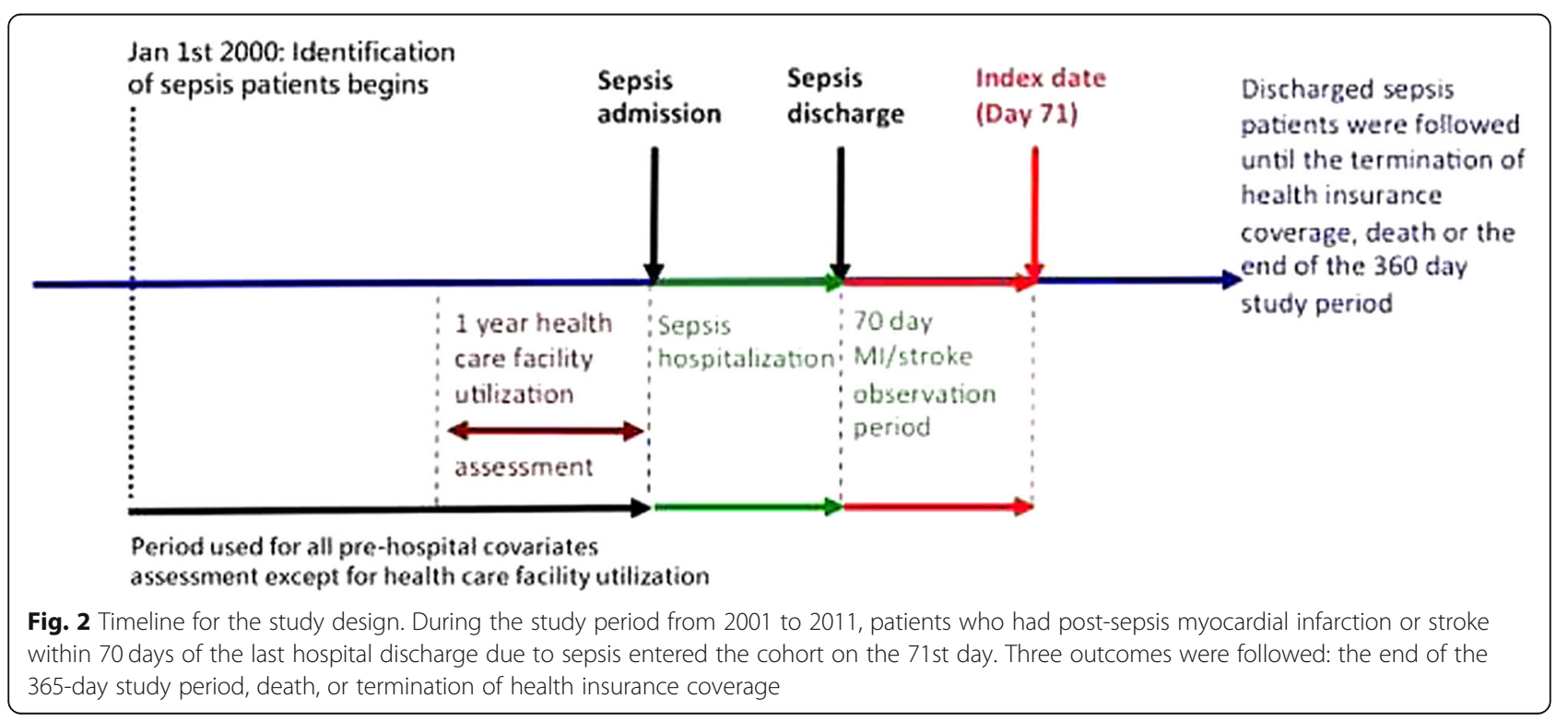


using the PS matching method mentioned above. To evaluate the success of the matching process, we calculated the standardized difference of matching covariates between the sepsis cohort and the non-sepsis comparison cohort. To evaluate the independent association between sepsis and post-sepsis $\mathrm{MI} /$ stroke, we calculated the incidence and 180-day mortality of MI/stroke among patients for the sepsis cohort and the PS-matched nonsepsis comparison cohort. We used univariate conditional logistic regression to evaluate the risk of incident $\mathrm{MI} /$ stroke and stratified Cox regression analysis to evaluate mortality risk. All statistical analyses were performed in SAS 9.4 (SAS Inc. Cary NC). In all analyses, a $P$ value of $<0.05$ was deemed significant.

\section{Results}

\section{Baseline characteristics of study patients}

From one million NHIRD participants, we identified 42, 316 patients who were hospitalized with sepsis (Fig. 1). There were 591 events observed in the 70-day risk period after discharge, of which $486(82.2 \%)$ were stroke, $108(18.3 \%)$ were $\mathrm{MI}$, and $3(0.5 \%)$ were concomitant stroke and MI. The preadmission characteristics of sepsis patients with/without post-sepsis MI/stroke are summarized in Table 1. Compared with patients without $\mathrm{MI} /$ stroke, patients who developed $\mathrm{MI} /$ stroke were older, associated with lower socioeconomic status, had a higher burden of preadmission comorbidities, and had a higher frequency of healthcare service utilization.

\section{Survival impact of post-sepsis acute $\mathrm{MI}$ and stroke}

The 180-day mortality for sepsis survivors without incident $\mathrm{MI} /$ stroke was $4.61 \%(1450 / 31,423)$. Patients who developed post-sepsis $\mathrm{MI} /$ stroke had twofold higher mortality compared to patients without post-sepsis MI/ stroke. The mortality of MI/stroke-naïve sepsis survivors rose to $7.35 \%(2309 / 31,423)$ at 365 days. The risk of mortality remained twofold higher for patients who developed post-sepsis MI/stroke. The crude mortality results are summarized in Table 2. To evaluate the survival impact of post-sepsis MI/stroke 180 days following hospital discharge, we plotted the cumulative hazard curve in Fig. 3. We observed that patients who developed MI/ stroke had a significantly higher cumulative mortality than patients without cardiovascular complications (Wilcoxon test $P=0.003$ ) within the 180 -day period. Adjusting for demographic variables and potential confounders (e.g., comorbidities) in the Cox proportional hazard model, we found a significant increase in 180-day mortality for patients with post-sepsis MI (hazard ratio [HR] 2.55, 95\% confidence interval [CI] 1.53, 4.27), postsepsis stroke (HR 2.19, 95\% CI 1.67, 2.87), and composite post-sepsis MI/stroke (HR 2.02, 95\% CI 1.65, 2.47) as compared with patients without post-sepsis $\mathrm{MI} /$ stroke
(Table 2). At 365 days, the increased risk persisted for patients with post-sepsis MI (HR 2.10, 95\% CI 1.34, 3.32), post-sepsis stroke (HR 2.02, 95\% CI 1.61, 2.52), and composite post-sepsis MI/stroke (HR 2.02, 95\% CI 1.66, 2.49). The full Cox model and effect estimates of the adjusted covariates are summarized in Table 3.

\section{Comparison between sepsis and non-sepsis cohorts}

Of the 42,316 identified sepsis patients, 41,251 were matched to a non-sepsis cohort by PS matching. The standardized difference of matching covariates between sepsis and non-sepsis patients was less than 6\%, indicating a successful match (Additional file 1: Table S1). We compared the incidence of post-sepsis $\mathrm{MI} /$ stroke between the sepsis and non-sepsis cohorts. For the sepsis cohort $(n=41,251)$, the incidence for MI, stroke, and composite $\mathrm{MI} /$ stroke, was $0.6 \%, 2.9 \%$, and $4.2 \%$, respectively. In contrast, for the non-sepsis cohort $(n=41,251)$, the incidence for MI, ischemic stroke, and composite $\mathrm{MI} /$ stroke, was $0.6 \%, 1.9 \%$, and $2.7 \%$, respectively. Compared to patients hospitalized without sepsis, patients hospitalized with sepsis had an increased risk for stroke (PS-matched OR 1.75, 95\% CI 1.60-1.92) and an increased risk for composite $\mathrm{MI} /$ stroke (PS-matched OR 1.72, 95\% CI 1.60-1.85), but not for MI alone (OR 1.01, 95\% CI 0.82-1.25) (Additional file 2: Table S2). Finally, we compared mortality between the two groups of patients. For the sepsis cohort $(n=41,251)$, the 180 -day mortality for MI, stroke, and composite $\mathrm{MI} /$ stroke was $13 \%, 9 \%, 10 \%$, respectively. On the other hand, for the non-sepsis cohort $(n=41,251)$, the 180 -day mortality for MI, stroke, and composite MI/stroke was $13 \%, 10 \%$, and $11 \%$, respectively. Cox regression showed sepsis was not associated with an increased hazard of mortality after MI, stroke, or MI/stroke (Additional file 3: Table S3).

\section{Discussion}

Sepsis is a major public health problem. Mortality associated with sepsis may be due to post-sepsis complications. In this comparison of patients hospitalized with sepsis and patients hospitalized without sepsis matched by PS, we showed that the increased risk of cardiovascular events after sepsis hospitalization is due to sepsis per se rather than a more generalized effect of acute illness, hospitalization, or deterioration in health status. We also showed that patients who developed cardiovascular events after sepsis hospitalization have an approximately twofold increase in 180-day or 365-day mortality as compared with patients who did not sustain cardiovascular events after sepsis hospitalization. However, survival after post-hospitalization $\mathrm{MI} /$ stroke did not differ between sepsis and non-sepsis patients.

Several major studies have shown that infection (e.g., pneumonia, sepsis) is associated with increased risk of 
Table 1 Characteristics of patients with and without post-sepsis MI/stroke

\begin{tabular}{|c|c|c|c|}
\hline Characteristics & $\begin{array}{l}\text { Patients who developed Ml/stroke, } \\
N=591\end{array}$ & $\begin{array}{l}\text { Patients who did not develop Ml/stroke, } \\
N=31,423\end{array}$ & $P$ value \\
\hline \multicolumn{4}{|l|}{ Demographics } \\
\hline Male sex & $374(63.28 \%)$ & $18,092(57.58 \%)$ & 0.0204 \\
\hline Age & $74.26 \pm 12.45$ & $66.11 \pm 19.13$ & $<.0001$ \\
\hline \multicolumn{4}{|l|}{ Urbanization level, \% } \\
\hline Level 1: urban area & $202(34.18 \%)$ & $12,058(38.37 \%)$ & \multirow[t]{4}{*}{0.1386} \\
\hline Level 2: metro area & $156(26.40 \%)$ & $8082(25.72 \%)$ & \\
\hline Level 3: suburban area & $159(26.90 \%)$ & $7409(23.58 \%)$ & \\
\hline Level 4: countryside area & $74(12.52 \%)$ & $3874(12.33 \%)$ & \\
\hline \multicolumn{4}{|l|}{ Insurance premium level, \% (New Taiwan dollars) } \\
\hline Dependent & $89(15.06 \%)$ & $4443(14.14 \%)$ & \multirow[t]{4}{*}{0.0044} \\
\hline No/poverty income level $(\$ 1-\$ 19,999)$ & $306(51.78 \%)$ & $14,281(45.45 \%)$ & \\
\hline Middle income level $(\$ 20,000-\$ 39,999)$ & $158(26.73 \%)$ & $10,107(32.17 \%)$ & \\
\hline High income level $(\geq \$ 40,000)$ & $38(6.43 \%)$ & $2592(8.25 \%)$ & \\
\hline \multicolumn{4}{|l|}{ Preadmission comorbidity, \% } \\
\hline Myocardial infarction & $41(6.94 \%)$ & $826(2.63 \%)$ & $<.0001$ \\
\hline Congestive heart failure & $126(21.32 \%)$ & 4691 (14.93\%) & $<.0001$ \\
\hline Peripheral vascular disease & $30(5.08 \%)$ & $1182(3.76 \%)$ & 0.0971 \\
\hline Cerebrovascular disease & $320(54.15 \%)$ & $7618(24.24 \%)$ & $<.0001$ \\
\hline Dementia & $77(13.03 \%)$ & $2818(8.97 \%)$ & 0.0006 \\
\hline Chronic pulmonary disease & $207(35.03 \%)$ & 9771 (31.1\%) & 0.0410 \\
\hline Rheumatologic disease & $10(1.69 \%)$ & $407(1.3 \%)$ & 0.3993 \\
\hline Peptic ulcer disease & $139(23.52 \%)$ & 7955 (25.32\%) & 0.3195 \\
\hline Mild liver disease & $66(11.17 \%)$ & $5950(18.94 \%)$ & $<.0001$ \\
\hline Diabetes without chronic complications & $236(39.93 \%)$ & $9013(28.68 \%)$ & $<.0001$ \\
\hline Diabetes with chronic complications & $91(15.4 \%)$ & $3263(10.38 \%)$ & $<.0001$ \\
\hline Hemiplegia or paraplegia & $44(7.45 \%)$ & $1198(3.81 \%)$ & $<.0001$ \\
\hline Renal disease & $82(13.87 \%)$ & $3930(12.51 \%)$ & 0.3196 \\
\hline Any malignancy, including leukemia and lymphoma & $46(7.78 \%)$ & $4822(15.35 \%)$ & $<.0001$ \\
\hline Moderate or severe liver disease & $8(1.35 \%)$ & 1047 (3.33\%) & 0.0076 \\
\hline Metastatic solid tumor & $7(1.18 \%)$ & $1300(4.14 \%)$ & 0.0003 \\
\hline AIDS/HIV & $2(0.34 \%)$ & $31(0.1 \%)$ & 0.0719 \\
\hline Alcohol/drug use & $8(1.35 \%)$ & $786(2.5 \%)$ & 0.0755 \\
\hline Psychiatric disorder & $112(18.95 \%)$ & $5341(17 \%)$ & 0.2106 \\
\hline Neurologic disorder & $82(13.87 \%)$ & $2612(8.31 \%)$ & $<.0001$ \\
\hline Obesity & $1(0.17 \%)$ & $94(0.3 \%)$ & 1.0000 \\
\hline Bed-ridden status & $45(7.61 \%)$ & $1226(3.9 \%)$ & $<.0001$ \\
\hline $\begin{array}{l}\text { Solid organ transplantation such as renal or heart } \\
\text { transplantation }\end{array}$ & $0(0 \%)$ & $45(0.14 \%)$ & 1.0000 \\
\hline \multicolumn{4}{|l|}{ Healthcare service utilization, \% } \\
\hline Number of OPD visits & $30.96 \pm 22.85$ & $29.95 \pm 22.79$ & 0.2851 \\
\hline Number of emergency department visits & $1.06 \pm 2.33$ & $0.85 \pm 2.02$ & 0.0116 \\
\hline Number of hospital admissions & $1.45 \pm 1.81$ & $1.30 \pm 1.88$ & 0.0563 \\
\hline
\end{tabular}

Summary and comparison of the demographics and underlying comorbidities of the study cohort stratified by post-sepsis Ml/stroke MI myocardial infarction, AIDS/HIV acquired immune deficiency syndrome/human immunodeficiency virus, OPD outpatient department 
Table 2 One hundred eighty- and 365-day mortality of patients with and without post-sepsis MI/stroke

\begin{tabular}{lllllll}
\hline Full sepsis cohort & 180 days & & & 365 days \\
\cline { 2 - 3 } & Mortality rate & $\begin{array}{l}\text { Confounder-adjusted } \\
\text { hazard ratio }\end{array}$ & & Mortality rate & $\begin{array}{l}\text { Confounder-adjusted } \\
\text { hazard ratio }\end{array}$ \\
\hline Patients with post-sepsis Ml & $13.89 \%(15 / 108)$ & $2.55(1.53,4.27)$ & & $17.59 \%(19 / 108)$ & $2.10(1.34,3.32)$ \\
Patients with post-sepsis stroke & $11.32 \%(55 / 486)$ & $2.19(1.67,2.87)$ & & $16.67 \%(81 / 486)$ & $2.02(1.61,2.52)$ \\
Patients with post-sepsis Ml or stroke & $11.68 \%(69 / 591)$ & $2.02(1.65,2.47)$ & & $16.75 \%(99 / 591)$ & $2.02(1.66,2.49)$ \\
Patients without post-sepsis complications & $4.61 \%(1450 / 31,423)$ & Reference & & $7.35 \%(2309 / 31,423)$ & Reference \\
\hline
\end{tabular}

Representation of the survival analysis results using Cox regression model summarizing survival impact of post-sepsis Ml, post-sepsis stroke, and post-sepsis MI/ stroke while accounting for potential confounders

$M I$ myocardial infarction

incident cardiovascular diseases in the short term and long term [4, 7]. Corrales-Medina et al. demonstrated that pneumonia was associated with increased shortterm and long-term risk of cardiovascular diseases in two large cohorts (the Cardiovascular Health Study and the Atherosclerosis Risk in Communities Study) [7]. Wang et al. compared sepsis and non-sepsis patients in a population-based study and corroborated CorralesMedina's findings, showing an increased long-term risk of coronary heart disease among patients surviving sepsis [4].

In this study, we further investigated a critical yet unresolved question: whether post-sepsis $\mathrm{MI} /$ stroke has an independent impact on the mid- and long-term survival among sepsis survivors. Consistent with the findings by Smilowitz et al., we showed post-sepsis MI/stroke itself is an independent risk factor for mortality among sepsis survivors. Smilowitz et al. showed that the in-hospital mortality is higher among patients who had both sepsis and MI as compared with patients who had sepsis alone (adjusted odds ratio 1.24; 95\% CI 1.22-1.26) [22]. However, the aforementioned study did not address the potential survivor bias [23]. We previously found the first 70 days after hospital discharge to be a period of increased susceptibility to incident cardiovascular events for sepsis patients. In addition, the mortality rate was high in the first few weeks after discharge from a sepsis hospitalization. Therefore, we indexed the cohort entry at day 70 after hospital discharge, thereby mitigating the influence of potential survivor bias or competing risk by early death. Wang et al. analyzed the impact of MI on sepsis survival, which was based on the same hospital stay. In contrast, using population-based longitudinal follow-up data, we were able to provide the evidence on the long-term outcome in patients associated with postsepsis cardiovascular events. As shown in the current study, cardiovascular events had a major adverse impact on long-term survival of sepsis patients, suggesting a need to monitor for cardiovascular events among sepsis survivors.

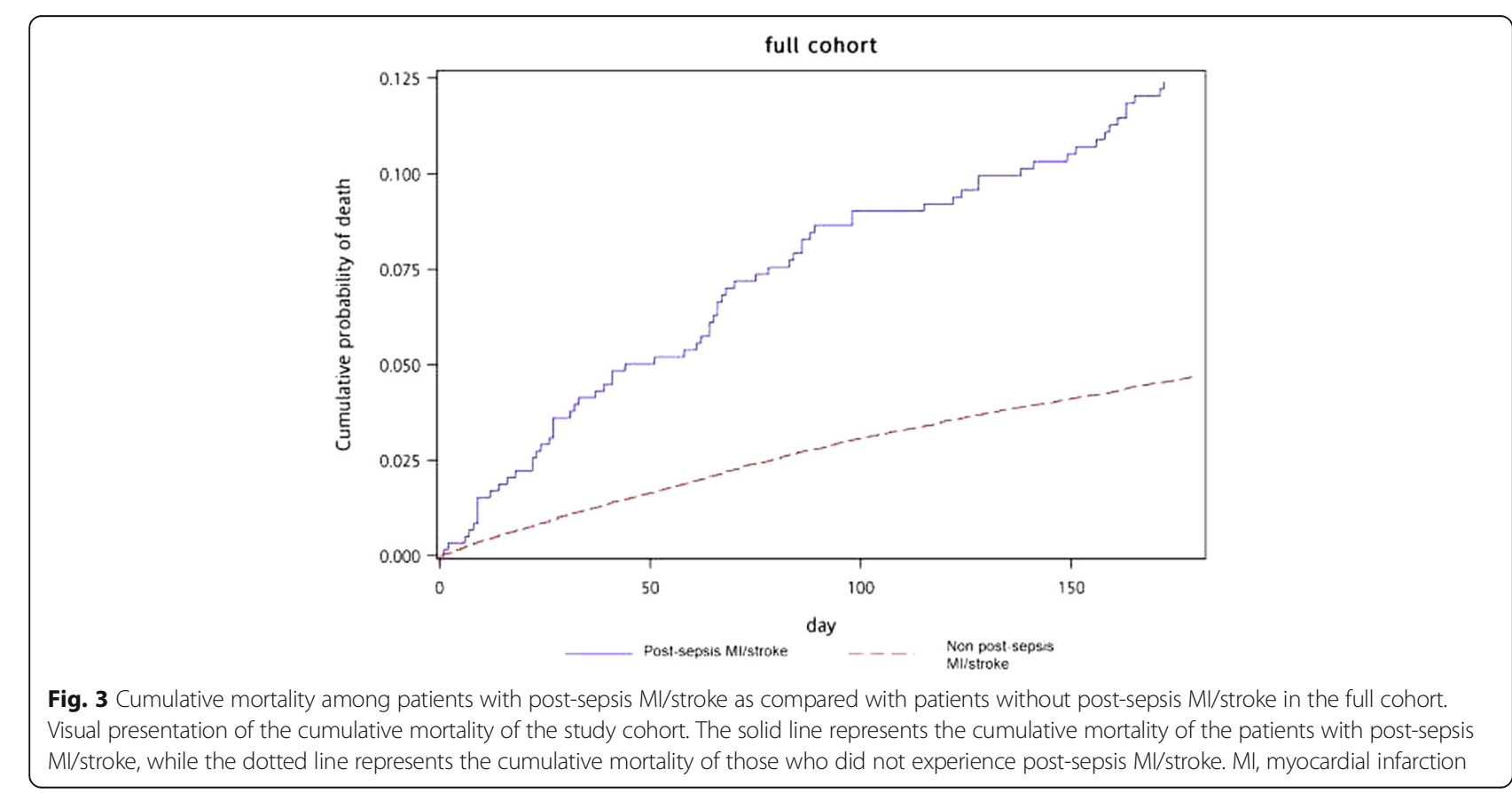


Table 3 Results of multivariable analysis showing the adjusted hazard ratio associated with the 180-day and 365-day mortality of each variable

\begin{tabular}{|c|c|c|c|c|}
\hline Characteristics & 180-day mortality, HR (95\% Cl) & $P$ value & 365-day mortality, HR (95\% Cl) & $P$ value \\
\hline Post-sepsis Ml/stroke & $2.02(1.65,2.47)$ & $<.0001$ & $2.02(1.65,2.47)$ & $<.0001$ \\
\hline \multicolumn{5}{|l|}{ Demographics } \\
\hline Male sex & $1.38(1.27,1.51)$ & $<.0001$ & $1.38(1.27,1.51)$ & $<.0001$ \\
\hline Age & $1.03(1.02,1.03)$ & $<.0001$ & $1.03(1.02,1.03)$ & $<.0001$ \\
\hline \multicolumn{5}{|l|}{ Urbanization level, \% } \\
\hline Level 1: urban area & $1.70(1.42,2.03)$ & $<.0001$ & $1.69(1.46,1.95)$ & $<.0001$ \\
\hline Level 2: metro area & $1.40(1.16,1.69)$ & 0.0005 & $1.45(1.25,1.69)$ & $<.0001$ \\
\hline Level 3: suburban area & $1.09(0.91,1.32)$ & 0.3524 & $1.10(0.94,1.27)$ & 0.2392 \\
\hline Level 4: countryside area & Reference & - & Reference & - \\
\hline \multicolumn{5}{|l|}{ Insurance premium level, \% (New Taiwan dollars) } \\
\hline Dependent & Reference & - & Reference & - \\
\hline No/poverty income level $(\$ 1-\$ 19,999)$ & $1.06(0.94,1.2)$ & 0.3171 & $1.06(0.94,1.2)$ & 0.3171 \\
\hline Middle income level $(\$ 20,000-\$ 39,999)$ & $1.25(1.1,1.44)$ & 0.001 & $1.25(1.1,1.44)$ & 0.001 \\
\hline High income level $(\geq \$ 40,000)$ & $0.84(0.68,1.04)$ & 0.1034 & $0.84(0.68,1.04)$ & 0.1034 \\
\hline \multicolumn{5}{|l|}{ Preadmission comorbidity, \% } \\
\hline Myocardial infarction & $1.03(0.83,1.28)$ & 0.7758 & $1.03(0.83,1.28)$ & 0.7758 \\
\hline Congestive heart failure & $1.16(1.04,1.29)$ & 0.0069 & $1.16(1.04,1.29)$ & 0.0069 \\
\hline Peripheral vascular disease & $0.90(0.74,1.1)$ & 0.319 & $0.90(0.74,1.10)$ & 0.319 \\
\hline Cerebrovascular disease & $1.16(1.06,1.28)$ & 0.0021 & $1.16(1.06,1.28)$ & 0.0021 \\
\hline Dementia & $1.47(1.31,1.65)$ & $<.0001$ & $1.47(1.31,1.65)$ & $<.0001$ \\
\hline Chronic pulmonary disease & $1.11(1.02,1.21)$ & 0.022 & $1.11(1.02,1.21)$ & 0.022 \\
\hline Rheumatologic disease & $1.27(0.91,1.78)$ & 0.1602 & $1.27(0.91,1.78)$ & 0.1602 \\
\hline Peptic ulcer disease & $0.96(0.88,1.06)$ & 0.4459 & $0.96(0.88,1.06)$ & 0.4459 \\
\hline Mild liver disease & $1.07(0.95,1.20)$ & 0.2567 & $1.07(0.95,1.20)$ & 0.2567 \\
\hline Diabetes without chronic complications & $1.09(0.99,1.20)$ & 0.0987 & $1.09(0.99,1.20)$ & 0.0987 \\
\hline Diabetes with chronic complications & $0.98(0.85,1.13)$ & 0.7508 & $0.98(0.85,1.13)$ & 0.7508 \\
\hline Hemiplegia or paraplegia & $1.56(0.44,5.47)$ & 0.4913 & $1.56(0.44,5.47)$ & 0.4913 \\
\hline Renal disease & $1.15(1.02,1.29)$ & 0.0184 & $1.15(1.02,1.29)$ & 0.0184 \\
\hline Any malignancy, including leukemia and lymphoma & $1.40(1.25,1.57)$ & $<.0001$ & $1.40(1.25,1.57)$ & $<.0001$ \\
\hline Moderate or severe liver disease & $1.72(1.40,2.10)$ & $<.0001$ & $1.72(1.40,2.10)$ & $<.0001$ \\
\hline Metastatic solid tumor & $1.19(0.99,1.44)$ & 0.0717 & $1.19(0.99,1.44)$ & 0.0717 \\
\hline AIDS/HIV & $2.05(0.66,6.39)$ & 0.2172 & $2.05(0.66,6.39)$ & 0.2172 \\
\hline Alcohol/drug use & $1.08(0.83,1.42)$ & 0.5569 & $1.08(0.83,1.42)$ & 0.5569 \\
\hline Psychiatric disorder & $0.96(0.86,1.07)$ & 0.4139 & $0.96(0.86,1.07)$ & 0.4139 \\
\hline Neurologic disorder & $1.03(0.90,1.18)$ & 0.6724 & $1.03(0.90,1.18)$ & 0.6724 \\
\hline Obesity & $0.75(0.28,2.00)$ & 0.567 & $0.75(0.28,2.00)$ & 0.567 \\
\hline Bed-ridden status & $0.76(0.22,2.66)$ & 0.6717 & $0.76(0.22,2.66)$ & 0.6717 \\
\hline Solid organ transplantation such as renal or heart transplantation & $1.26(0.4,3.92)$ & 0.6922 & $1.26(0.40,3.92)$ & 0.6922 \\
\hline \multicolumn{5}{|l|}{ Healthcare service utilization, \% } \\
\hline Number of OPD visits & $1.00(1.00,1.00)$ & 0.9105 & $1.00(1.00,1.00)$ & 0.9105 \\
\hline Number of emergency department visits & $1.02(1.01,1.04)$ & 0.0002 & $1.02(1.01,1.04)$ & 0.0002 \\
\hline Number of hospital admissions & $1.08(1.06,1.10)$ & $<.0001$ & $1.08(1.06,1.10)$ & $<.0001$ \\
\hline
\end{tabular}


The relatively low 180-day and 365-day mortality in this cohort as compared with previous reports is worth the discussion. Compared with our post-sepsis MI mortality (180-day mortality, $13.89 \%$; 365-day mortality, $17.59 \%)$, Smilowitz et al. showed the in-hospital all-cause mortality for patients with sepsis and MI is 35.8\% [22]. A similar observation was made for mortality not induced by post-sepsis MI/stroke: our 180-day (4.61\%) and 356day $(7.35 \%)$ mortalities are lower than the in-hospital mortality (16.8\%) reported by Smilowitz et al. Likewise, Weycker et al. and Braun et al. found post-sepsis 1-year all-cause mortality to be $51.4 \%$ and $36.1 \%$, respectively $[24,25]$. The relatively low mortality observed in our cohort could be explained by the different index dates between the current studies and previous studies. Our study design built in a lag time between hospital discharge and index date, which prevents us from gaging the high mortality of sepsis patients within this critical 70-day window. The mortality of this cohort in the 70-day period after hospital discharge was as high as $24.3 \%$. The exclusion of patients who died within 70 days of discharge resulted in a low mortality rate in our patients.

The findings of this study support the hypothesis that the long-term mortality of sepsis is at least partially mediated through post-sepsis MI/stroke. The observed adverse survival impact of post-sepsis $\mathrm{MI} /$ stroke implicates the potential benefits from early preventative measures. Among the potential mediators of post-sepsis cardiovascular complications [26-31], platelets might serve as a major target for pharmacological intervention [32-36]. The protective role of long-term aspirin use in the risk of cardiovascular events is well documented [37-40], justifying future trials on its preventive role in the cardiovascular events after a sepsis episode. Such a trial may also consider including sepsis survivors without traditional cardiac risk factors. Another promising class of pharmacologic preventive agents is statins, which also have a well-established established role in the prevention of cardiovascular events. Aside from lipid-lowering properties, the pleiotropic effects of statins including anti-inflammatory and recently discovered bactericidal effects may offer added benefit to sepsis survivors [41, 42]. Definitive roles of these medications for sepsis patients awaits further investigation.

Our study has several strengths. The National Taiwan Health Insurance Database is large and comprehensive and has lent itself to many longitudinal analyses of sepsis. We used the Angus System for sepsis case identification, which is the best available algorithm for an administrative database. We used Cox regression and limited to cardiovascular events within 70 days of the sepsis event to mitigate concerns about survival time bias.

The results of this study should be interpreted in light of a few limitations. First, like other administrative databases, certain important risk factors for cardiovascular disease such as body mass index, smoking status, and alcohol consumption are lacking. Second, reverse causation may be at play given that MI or cerebrovascular events might both predispose patients to sepsis and impact the patients' survival. To avoid the potential reverse causation, post-sepsis MI/stroke was ascertained after the hospital discharge from sepsis to establish temporality, and multivariable analyses were used to adjust for confounding effects introduced by prior myocardial infarction or cerebrovascular events. Third, with respect to our finding that there was no association between sepsis and subsequent MI, two potential causes may underlie this phenomenon. First, the comparison cohort consisted of hospitalized patients, who were already at elevated risk of MI, rather than healthy population controls. Second, the number of patients with incident MI in the sepsis cohort and the comparison cohort was only 251 and 228, respectively. As such, we did not have sufficient statistical power to detect this subtle difference.

\section{Conclusions}

Sepsis is a major public health problem, and mortality associated with sepsis may be due to the complications that develop or accelerate after the sepsis episode. We demonstrated that the increased risk of cardiovascular events after sepsis is due to sepsis per se rather than a more generalized effect of deteriorating health status. Incident cardiovascular events after sepsis have a strong adverse impact on long-term survival. Current work may inform the need to extend sepsis care from acute stage management to the prevention of cardiovascular complications in the convalescent stage.

\section{Additional files}

Additional file 1: Table S1. Characteristics of sepsis patients and propensity score-matched non-sepsis patients. Summary and comparison of the demographics and underlying comorbidities of the sepsis cohort and the non-sepsis cohort. Abbreviations: MI, myocardial infarction; AIDS/ HIV, acquired immune deficiency syndrome/human immunodeficiency virus. (DOCX $17 \mathrm{~kb})$

Additional file 2: Table S2. Comparison of incidence (events/1000 person) of MI/stroke in sepsis and propensity score-matched non-sepsis cohort. Representation of the multivariable logistic regression analysis summarizing the association between sepsis and incidence of Ml, stroke and $\mathrm{Ml} /$ stroke while accounting for potential confounders. Abbreviations: MI, myocardial infarction; PS, propensity score; OR, odds ratio. (DOCX 15 kb)

Additional file 3: Table S3. Comparison of mortality of $\mathrm{Ml} /$ stroke in sepsis and propensity score-matched non-sepsis cohorts. Representation of the survival analysis results using Cox regression model summarizing survival impact of sepsis on the mortality of $\mathrm{Ml}$, stroke and $\mathrm{MI}$ /stroke while accounting for potential confounders. Abbreviations: MI, myocardial infarction; HR, hazard ratio. (DOCX $15 \mathrm{~kb}$ )

Additional file 4: Appendix 1. Codes associated with infection. The ICD-9-CM codes utilized to identify infections. Appendix 2. Codes associated with organ dysfunction. The ICD-9-CM codes utilized to identify organ dysfunction. (DOCX 29 kb) 


\section{Abbreviations}

ED: Emergency department; HR: Hazard ratio; ICD: International Classification of Diseases; MI: Myocardial infarction; NHIRD: National Health Insurance Research Database

\section{Acknowledgements}

We thank the staff of the Core Labs at the Department of Medical Research at National Taiwan University Hospital for the technical support, Medical Wisdom Consulting Group for the technical assistance in statistical analysis, and National Taiwan University Hospital Health Economics and Outcome Research Group for the advice on study design.

\section{Authors' contributions}

CCL and SSC had full access to all the data in the study and take responsibility for the integrity of the data and the accuracy of the data analysis. CCL was responsible for the concept and design and obtained funding. MHW, YHW, SSC, PYT, CCL, and MGL drafted the manuscript. CCL, PYT, MGL, SHL, CCTC, SSC, JYW, and JRH critically revised the manuscript for important intellectual content. WCL was responsible for the statistical analysis. CCL and SSC were responsible for the supervision. All authors read and approved the final manuscript.

\section{Funding}

This study is supported by the Taiwan National Science Foundation Grant NSC 102-2314-B-002-131-MY3; Taiwan National Ministry of Science and Technology Grants MOST 104-2314-B-002-039-MY3, and MOST 105-2811-B-002031. No funding bodies had any role in the study design, data collection and analysis, decision to publish, or preparation of the manuscript.

\section{Availability of data and materials}

The datasets generated and/or analyzed during the current study are not publicly available due to the data confidentiality requirements of the ethics committee but can be made available by the corresponding author on reasonable request and approval from the ethics committee.

\section{Ethics approval and consent to participate}

National Health Insurance Research Database is a de-identified database, and its use in the current work has been approved by the Institutional Review Board of National Taiwan University Hospital.

\section{Consent for publication}

Not applicable

\section{Competing interests}

The authors declare that they have no competing interests.

\section{Author details}

'Department of Emergency Medicine, Chang Gung Memorial Hospital, Kaohsiung, Taiwan and Chang Gung University College of Medicine, Taoyuan, Taiwan. ${ }^{2}$ Department of Epidemiology, Bloomberg School of Public Health, Johns Hopkins University, Baltimore, MD, USA. ${ }^{3}$ Department of Emergency Medicine, National Taiwan University Hospital, No.7, Chung Shan S. Rd., Zhongzheng Dist., Taipei 100, Taiwan. ${ }^{4}$ Canberra Hospital, ACT Health, Canberra, Australian Capital Territory, Australia. ${ }^{5}$ Department of Rehabilitation and Physical Medicine, Taipei Veterans General Hospital, Taipei, Taiwan. ${ }^{6}$ Department of Medicine, College of Medicine, National Yang Ming University, Taipei, Taiwan. ${ }^{7}$ Department of Emergency Medicine, Chang Gung Memorial Hospital, Keelung, Taiwan and Chang Gung University College of Medicine, Taoyuan, Taiwan. ${ }^{8}$ Department of Family Medicine, Taipei Medical University Hospital and School of Medicine, Taipei Medical University, Taipei, Taiwan. ${ }^{9}$ Department of Emergency Medicine, Health Data Science Research Group, National Taiwan University Hospital, No.7, Chung Shan S. Rd.,

Zhongzheng Dist., Taipei 100, Taiwan.

\section{Received: 13 February 2019 Accepted: 21 August 2019}

\section{Published online: 02 September 2019}

\section{References}

1. Singer M, Deutschman CS, Seymour CW, Shankar-Hari M, Annane D, Bauer $M$, et al. The Third International Consensus Definitions for Sepsis and Septic Shock (Sepsis-3). Jama. 2016;315(8):801-10.
2. Lee CC, Yo CH, Lee MG, Tsai KC, Lee SH, Chen YS, et al. Adult sepsis - a nationwide study of trends and outcomes in a population of 23 million people. J Infection. 2017;75(5):409-19.

3. Prescott HC, Osterholzer JJ, Langa KM, Angus DC, Iwashyna TJ. Late mortality after sepsis: propensity matched cohort study. BMJ. 2016;353:12375.

4. Wang HE, Moore JX, Donnelly JP, Levitan EB, Safford MM. Risk of acute coronary heart disease after sepsis hospitalization in the REasons for Geographic and Racial Differences in Stroke (REGARDS) Cohort. Clin Infect Dis. 2017;65(1):29-36.

5. Ou SM, Chu H, Chao PW, Lee YJ, Kuo SC, Chen TJ, et al. Long-term mortality and major adverse cardiovascular events in sepsis survivors: a nationwide population-based study. Am J Respir Crit Care Med. 2016;194(2):209-17.

6. Jafarzadeh SR, Thomas BS, Warren DK, Gill J, Fraser VJ. Longitudinal study of the effects of bacteremia and sepsis on 5-year risk of cardiovascular events. Clin Infect Dis. 2016;63(4):495-500.

7. Corrales-Medina VF, Alvarez KN, Weissfeld LA, Angus DC, Chirinos JA, Chang CC, et al. Association between hospitalization for pneumonia and subsequent risk of cardiovascular disease. JAMA. 2015;313(3):264-74.

8. Kaynar AM, Yende S, Zhu L, Frederick DR, Chambers R, Burton CL, et al. Effects of intra-abdominal sepsis on atherosclerosis in mice. Crit Care. 2014;18(5):469.

9. Corrales-Medina VF, Madjid M, Musher DM. Role of acute infection in triggering acute coronary syndromes. Lancet Infect Dis. 2010;10(2):83-92.

10. Carson WF, Cavassani KA, Dou Y, Kunkel SL. Epigenetic regulation of immune cell functions during post-septic immunosuppression. Epigenetics. 2011;6(3):273-83.

11. Lai CC, Lee MG, Lee WC, Chao CC, Hsu TC, Lee SH, et al. Susceptible period for cardiovascular complications in patients recovering from sepsis. CMAJ. 2018;190(36):E1062-E9.

12. Kaukonen KM, Bailey M, Suzuki S, Pilcher D, Bellomo R. Mortality related to severe sepsis and septic shock among critically ill patients in Australia and New Zealand, 2000-2012. Jama. 2014;311(13):1308-16.

13. Walkey AJ, Wiener RS, Lindenauer PK. Utilization patterns and outcomes associated with central venous catheter in septic shock: a population-based study. Crit Care Med. 2013;41(6):1450-7.

14. Uno H, Wittes J, Fu H, Solomon SD, Claggett B, Tian L, et al. Alternatives to hazard ratios for comparing the efficacy or safety of therapies in noninferiority studies. Ann Intern Med. 2015;163(2):127-34.

15. Uno H, Claggett B, Tian L, Inoue E, Gallo P, Miyata T, et al. Moving beyond the hazard ratio in quantifying the between-group difference in survival analysis. J Clin Oncol. 2014;32(22):2380-5.

16. von Elm E, Altman DG, Egger M, Pocock SJ, Gotzsche PC, Vandenbroucke $J$, et al. Strengthening the Reporting of Observational Studies in Epidemiology (STROBE) statement: guidelines for reporting observational studies. BMJ. 2007;335(7624):806-8.

17. Angus DC, Linde-Zwirble WT, Lidicker J, Clermont G, Carcillo J, Pinsky MR. Epidemiology of severe sepsis in the United States: analysis of incidence, outcome, and associated costs of care. Crit Care Med. 2001;29(7):1303-10.

18. Charlson M, Szatrowski TP, Peterson J, Gold J. Validation of a combined comorbidity index. J Clin Epidemiol. 1994;47(11):1245-51.

19. Cheng CL, Kao YH, Lin SJ, Lee CH, Lai ML. Validation of the National Health Insurance Research Database with ischemic stroke cases in Taiwan. Pharmacoepidemiol Drug Saf. 2011;20(3):236-42.

20. Cheng $\mathrm{CL}$, Lee $\mathrm{CH}$, Chen PS, Li YH, Lin SJ, Yang YH. Validation of acute myocardial infarction cases in the national health insurance research database in Taiwan. J Epidemiol. 2014;24(6):500-7.

21. Liu C, Hung Y, Chuang Y. Incorporating development stratification of Taiwan townships into sampling design of large scale health interview survey. J Health Manag. 2005;4(1):1-22.

22. Smilowitz NR, Gupta N, Guo Y, Bangalore S. Comparison of outcomes of patients with sepsis with versus without acute myocardial infarction and comparison of invasive versus noninvasive management of the patients with infarction. Am J Cardiol. 2016;117(7):1065-71.

23. Zhou Z, Rahme E, Abrahamowicz M, Pilote L. Survival bias associated with time-to-treatment initiation in drug effectiveness evaluation: a comparison of methods. Am J Epidemiol. 2005;162(10):1016-23.

24. Weycker D, Akhras KS, Edelsberg J, Angus DC, Oster G. Long-term mortality and medical care charges in patients with severe sepsis. Crit Care Med. 2003;31(9):2316-23.

25. Braun L, Riedel AA, Cooper LM. Severe sepsis in managed care: analysis of incidence, one-year mortality, and associated costs of care. J Manag Care Pharm. 2004;10(6):521-30. 
26. Kilickap M, Goksuluk H, Candemir B, Kaya CT, Ozcan OU, Turhan S, et al. Evaluation of acute infection-induced endothelial dysfunction and its potential mediators. Acta Cardiol. 2011;66(5):581-7.

27. Levi M. Platelets in sepsis. Hematology (Amsterdam, Netherlands). 2005; 10(Suppl 1):129-31.

28. Merx MW, Weber C. Sepsis and the heart. Circulation. 2007;116(7):793-802.

29. Okamoto K, Tamura T, Sawatsubashi Y. Sepsis and disseminated intravascular coagulation. J Intensive Care. 2016;4:23.

30. Vallance P, Collier J, Bhagat K. Infection, inflammation, and infarction: does acute endothelial dysfunction provide a link? Lancet. 1997;349(9062):1391-2.

31. Zanotti-Cavazzoni SL, Hollenberg SM. Cardiac dysfunction in severe sepsis and septic shock. Curr Opin Crit Care. 2009;15(5):392-7.

32. Thomas MR, Storey RF. The role of platelets in inflammation. Thromb Haemost. 2015;114(3):449-58.

33. Semple JW, Italiano JE Jr, Freedman J. Platelets and the immune continuum. Nat Rev Immunol. 2011;11(4):264-74.

34. Semple JW, Freedman J. Platelets and innate immunity. Cell Mol Life Sci. 2010;67(4):499-511.

35. Russwurm S, Vickers J, Meier-Hellmann A, Spangenberg P, Bredle D, Reinhart $\mathrm{K}$, et al. Platelet and leukocyte activation correlate with the severity of septic organ dysfunction. Shock. 2002;17(4):263-8.

36. Katz JN, Kolappa KP, Becker RC. Beyond thrombosis: the versatile platelet in critical illness. Chest. 2011;139(3):658-68

37. Arnett DK, Blumenthal RS, Albert MA, Michos ED, Buroker AB, Miedema MD, et al. 2019 ACC/AHA guideline on the primary prevention of cardiovascular disease. J Am Coll Cardiol. 2019;26029. https://doi.org/10.1161/CIR. 0000000000000678.

38. Thrombosis prevention trial: randomised trial of low-intensity oral anticoagulation with warfarin and low-dose aspirin in the primary prevention of ischaemic heart disease in men at increased risk. The Medical Research Council's General Practice Research Framework. Lancet. 1998; 351(9098):233-41.

39. Ridker PM, Cook NR, Lee IM, Gordon D, Gaziano JM, Manson JE, et al. A randomized trial of low-dose aspirin in the primary prevention of cardiovascular disease in women. N Engl J Med. 2005;352(13):1293-304.

40. de Gaetano G, Collaborative Group of the Primary Prevention P. Low-dose aspirin and vitamin $\mathrm{E}$ in people at cardiovascular risk: a randomised trial in general practice. Collaborative Group of the Primary Prevention Project. Lancet. 2001;357(9250):89-95.

41. Lee MG, Lee CC, Lai CC, Hsu TC, Porta L, Lee M, et al. Preadmission statin use improves the outcome of less severe sepsis patients - a population-based propensity score matched cohort study. Br J Anaesth. 2017;119(4):645-54.

42. Lee CC, Lee MG, Hsu TC, Porta L, Chang SS, Yo CH, et al. A populationbased cohort study on the drug-specific effect of statins on sepsis outcome. Chest. 2018;153(4):805-15.

\section{Publisher's Note}

Springer Nature remains neutral with regard to jurisdictional claims in published maps and institutional affiliations. 